\title{
GESTIÓN ÓPTIMA DE REDES DE RIEGO MEDIANTE EL CONTROL DE LA OPERACIÓN DE LOS PUNTOS CRÍTICOS
}

\author{
Fernández García, $I^{1}{ }^{1}$, González Perea, ${ }^{1}{ }^{1}$, Rodríguez Díaz, J. $A^{1}$., Moreno, \\ M.A. ${ }^{2}$, Montesinos, $P{ }^{1}{ }^{1}$, Camacho Poyato, $E .^{1}$
}

\begin{abstract}
1 Departamento de Agronomía. Universidad de Córdoba. Campus Rabanales, Edif. Da Vinci, 14071. Córdoba. E-mail: g52fegai@uco.es,g72goper@uco.es, jarodriguez@uco.es, pmontesinos@uco.es, ecamacho@uco.es
\end{abstract}

2 Centro Regional de Estudios del Agua, Universidad de Castilla-La Mancha, 02071, Albacete. E-mail: miguelangel.moreno@uclm.es

\section{Resumen}

La necesidad de disminuir los costes energéticos que los agricultores tienen que afrontar tras la modernización de los sistemas de distribución del agua, está llevando a un aumento de la demanda de estrategias encaminadas a reducir el consumo de este recurso. En este trabajo se propone una metodología fundamentada en la optimización de la altura manométrica en la estación de bombeo y el control de la operación de los puntos críticos con el fin de disminuir la demanda energética de la red, asegurando una presión mínima en los hidrantes operativos. La estrategia considera dos escenarios de gestión: escenario $A$, en el que se determina la altura manométrica óptima considerando un $100 \%$ de simultaneidad de hidrantes; y escenario $B$, en el que se determina la altura manométrica óptima cuando los hidrantes críticos (identificados previamente) se desactivan. La metodología se ha aplicado en los 11 sectores que componen la comunidad de regantes del Bembézar Margen Derecha, determinando una reducción en la altura manométrica óptima entre los escenarios A y $B$ de $12 \mathrm{~m}$ y $13 \mathrm{~m}$ en dos sectores seleccionados como representativos. Esta reducción en la altura manométrica se traduce en un ahorro energético potencial de $23.2 \%$ y $25.8 \%$ en los sectores analizados. La adopción del escenario $A$ en las horas en las que el precio de la energía es mayor y la aplicación del escenario B sin restricciones horarias pueden suponer importantes ahorros en el coste energético.

\section{1-Introducción}

Según la FAO, la escasez de agua se produce cuando "la demanda supera el suministro de agua dulce en un área de determinada" (FAO 2013). Teniendo en cuenta que la población mundial continúa aumentando y se espera que alcance los 9000 millones de personas en 2050, es indiscutible que la presión sobre los recursos hídricos se incrementará.

Entre los sectores económicos, la agricultura es responsable del $70 \%$ de las extracciones de agua dulce en el mundo (FAO 2013). Mediante la expansión de la agricultura de regadío, se ha conseguido aumentar y garantizar las producciones agrícolas en muchas regiones. De esta forma, el riego tendrá un papel clave a la hora de asegurar la producción de alimentos para ese aumento de población. Se estima que para satisfacer la demanda de la población en 2050 , la producción de alimentos tendrá que aumentar un $60 \%$ (FAO 2013). Por tanto, la agricultura tendrá que adaptarse para satisfacer esa mayor demanda con un recurso cada vez más escaso. 
Una de las actuaciones que se han llevado a cabo para mejorar la gestión de este recurso ha consistido en la transformación de las infraestructuras de distribución del agua mediante canales abiertos hacia redes a presión, lo que ha supuesto un aumento de la eficiencia en la distribución del agua (MAPA 2001, MARM 2006). Por otro lado, las nuevas redes a presión han favorecido el cambio hacia sistemas de riego más eficientes, como el goteo o la aspersión, que implican una mejora adicional de la gestión del agua aumentando la eficiencia de aplicación en parcela (Carrillo Cobo et al. 2011). Sin embargo, este proceso de modernización ha traído consigo un aumento de las necesidades energéticas, lo que unido al incremento del precio de este recurso, justifica la necesidad de desarrollar estrategias encaminadas a mejorar la gestión de este recurso.

Varios autores han desarrollado estrategias que permiten mejorar la eficiencia energética en las redes de riego a presión. Algunas de ellas se fundamentan en la gestión de la red de riego en sectores mediante el establecimiento de turnos de riego (Jiménez Bello et al. 2010), en el control de la operación de los puntos críticos (Rodríguez Díaz et al. 2012) o en la optimización del funcionamiento de la estación de bombeo (Moreno et al. 2007). Mediante la aplicación de estas medidas, se han alcanzado ahorros energéticos que oscilan entre el $10 \%$ y el $36 \%$ en las redes de riego analizadas.

El impacto del aumento de las tarifas energéticas en la factura eléctrica es cada vez mayor por lo que en otros trabajos se ha considerado la optimización de la gestión de la red de riego teniendo en cuenta, además, la optimización del coste energético mediante la incorporación de la tarifa energética (Fernández García et al. 2014).

En este trabajo se propone una nueva estrategia de gestión de redes de riego, enfocada a la disminución del consumo de energía, fundamentada en la restricción de las horas disponibles para riego en los puntos críticos en periodos de tarificación punta.

\section{2- Metodología}

\section{1.- Zona de estudio}

La comunidad de regantes Bembézar Margen Derecha se sitúa entre las provincias de Sevilla y Córdoba y cuenta con una superficie regada de aproximadamente 12.000 ha, en las que en torno al $50 \%$ se dedican al cultivo de cítricos. Los sistemas de distribución del agua en esta comunidad de regantes se han modernizado en los últimos años y actualmente posee una red de riego a presión que abastece a 11 sectores independientes, organizados a la demanda (Figura 1).

La red se diseñó para suministrar $1,25 \mathrm{l} \cdot \mathrm{s}^{-1} \cdot \mathrm{ha}^{-1}$ con una presión mínima de funcionamiento en hidrante de 35 m.c.a. En la actualidad, las estaciones de bombeo de sus 11 sectores operan mediante una regulación dinámica de presión y las bombas se activan secuencialmente con el fin de mantener una presión de consigna. A la salida de cada estación de bombeo se encuentra un filtro cuyas pérdidas de carga son de 5 m.c.a. 


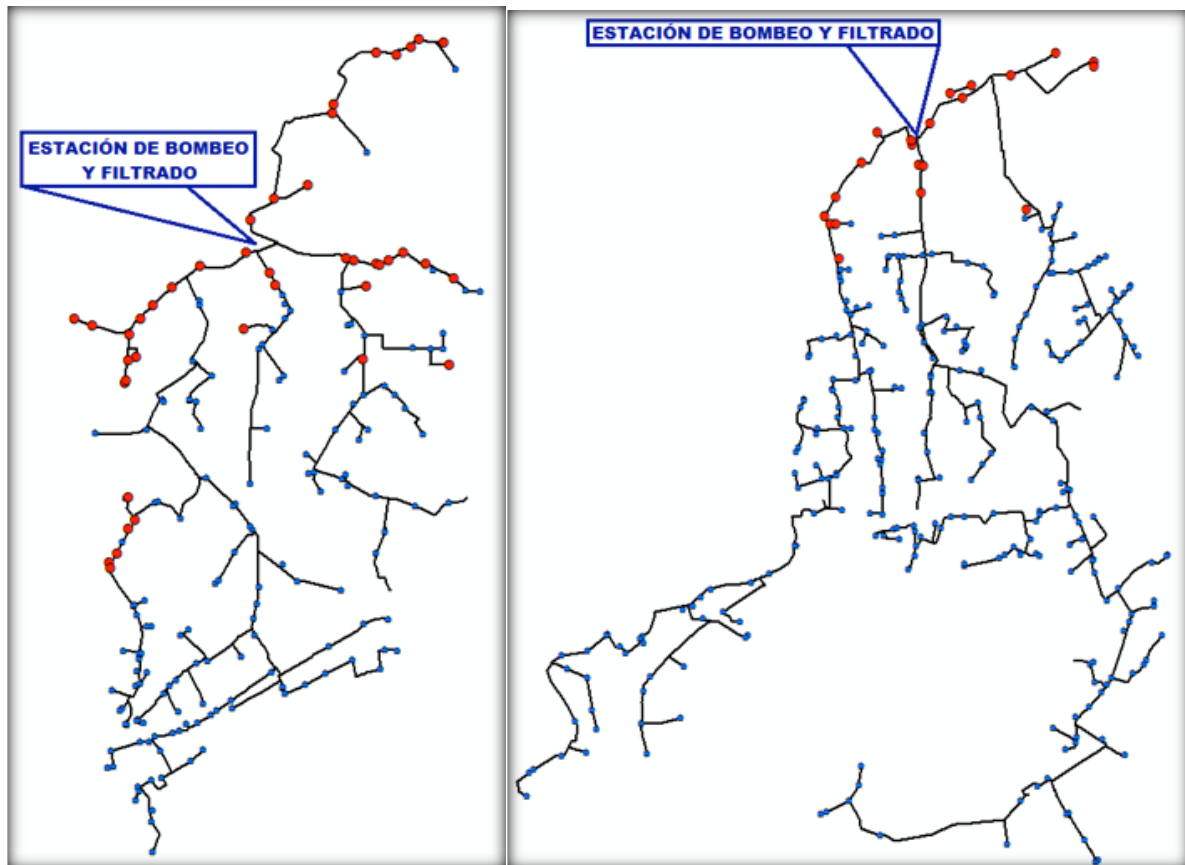

Figura 1. Representación esquemática de las redes de distribución de dos sectores (IX (izquierda) y X (derecha)), seleccionados para el análisis de resultados.

\section{2.- Escenarios de gestión}

Dos escenarios de gestión de redes de riego se han considerado para reducir la demanda de potencia, $\mathrm{P}(\mathrm{kW})$, (ecuación 1$)$ de la red de distribución de agua:

$$
\mathrm{P}=\frac{1}{1000} \cdot \frac{1}{\eta} \cdot \gamma \cdot \mathrm{Q} \cdot \mathrm{H}
$$

Siendo $\eta$ el rendimiento de la estación de bombeo, $\gamma$ el peso específico del agua $\left(\mathrm{Nm}^{-3}\right), Q$ el caudal demandado por la red $\left(\mathrm{m}^{3} \mathrm{~s}^{-1}\right)$ y $H$ la altura manométrica necesaria para satisfacer la presión de servicio en todos los hidrantes $(\mathrm{m})$

- Escenario A. Determinación del funcionamiento óptimo de la estación de bombeo considerando que todos los hidrantes riegan a la vez, garantizando una presión mínima de 35 m.c.a en cada hidrante.

- Escenario B. Optimización del funcionamiento de la estación de bombeo considerando que todos los hidrantes, excepto aquéllos identificados como puntos críticos, riegan simultáneamente. Los hidrantes críticos son aquellos que bien por su altura o su distancia con respecto a la estación de bombeo poseen requerimientos de energía elevados. Este escenario se fundamenta en evitar que los hidrantes críticos rieguen durante las horas en las que el precio de la energía es mayor. Al igual que en el escenario $A$, se garantiza una presión de 35 m.c.a. en todos los hidrantes que no actúan como críticos.

\section{3.- Modelización de la estación de bombeo}

La modelización de estaciones de bombeo consiste en simular el comportamiento de la estación de bombeo en distintos escenarios de manejo de la misma. Estos escenarios de 
manejo son, principalmente, la presión de consigna de la estación de bombeo y la secuencia de activación de bombas de velocidad fija y velocidad variable.

Con este modelo de simulación se puede determinar el tipo de regulación de la estación de bombeo que minimiza el consumo energético, y por lo tanto, el coste de operación del sistema, garantizando las condiciones de demanda de la red de riego.

El modelo de simulación de estaciones de bombeo requiere la siguiente información:

- Curvas características de las distintas bombas que componen la estación de bombeo, obtenidas de los catálogos de las bombas.

- Número de bombas activadas con un variador de velocidad y número de bombas fijas, según la instalación existente.

- Secuencia de activación de bombas variables y fijas, según la secuencia actual o variando la frecuencia para minimizar el consumo energético global.

- Rendimiento del motor de las bombas. En caso de no tener datos a este respecto, se pueden obtener rendimientos de bombas de semejante potencia y calidad.

- Rendimiento del variador de velocidad que activan las bombas de velocidad variable, quizá el parámetro más difícil de obtener en una estación de bombeo por la dificultad en la medida aguas arriba y aguas abajo del variador, con equipos de alta precisión y con necesidad de sincronismo de los equipos. Para considerar este rendimiento, se pueden utilizar curvas de rendimiento estandarizadas dependiendo de la potencia de la bomba y la capacidad del propio variador.

A partir de los datos de entrada, se ha desarrollado un modelo de simulación de estaciones de bombeo que reproduce el comportamiento de todas las bombas que conforman la estación para diferentes secuencias de activación. El modelo tiene en cuenta un intervalo para el caudal suministrado de $25 \mathrm{I} \mathrm{min}^{-1}$ y la presión necesaria para cada valor de caudal. Por último, para cada par de valores Q-H se determina la eficiencia de bombeo en función de la secuencia de activación de las bombas.

El resultado del modelo es una matriz en la que se puede determinar el rendimiento de la estación de bombeo para cada binomio Q-H y para cualquier tipo de regulación y presión de consigna (matriz Q-H-n). El modelo de simulación de la estación de bombeo se ha desarrollado en MaTLab (Pratap 2010).

\section{4.- Optimización del funcionamiento de la estación de bombeo}

A partir de la matriz Q-H-n obtenida mediante la modelización de la estación de bombeo, este algoritmo permite determinar para cada caudal (condicionado por el número de hidrantes abiertos), la altura manométrica y la eficiencia de bombeo que minimiza la ecuación 1 y, al mismo tiempo, garantiza la presión de servicio en hidrante. La demanda base de cada hidrante, $q_{h}\left(\mathrm{Is}^{-1}\right)$, se determina mediante la siguiente expresión:

$$
q_{h}=q_{s} \cdot S_{h}
$$

Siendo $q_{s}$ el caudal de diseño del hidrante $\left(1.2 \mathrm{Is}^{-1} \mathrm{ha} \mathrm{A}^{-1}\right)$ y $S_{h}$ (ha) la superficie asociada a cada hidrante. escenarios:

El algoritmo se ha desarrollado en MaTLab, diferenciando entre los siguientes 
- Escenario A. En este escenario se determina la presión mínima en la estación de bombeo que garantiza la presión de servicio en todos los hidrantes, considerando la operación simultánea de los mismos. La demanda base de cada hidrante se introduce en un modelo hidráulico de la red, el simulador EPANET (Rossman 2000), obteniendo de esta simulación la altura manométrica necesaria en la estación de bombeo para satisfacer la presión de servicio en todos los hidrantes. El caudal demandado en la red (suma de la demanda base de todos los hidrantes) y la altura manométrica obtenida mediante el simulador hidráulico son datos de entrada en la matriz Q-H-n. Como resultado, la matriz proporciona la altura manométrica que implica una demanda de potencia mínima y que garantiza el caudal demandado y la presión requerida en hidrante. La altura manométrica obtenida en la matriz será siempre igual o mayor a la altura manométrica obtenida en el simulador hidráulico.

- Escenario B. El análisis del escenario B contempla varias etapas. En primer lugar y al igual que en el escenario A, se parte de la operación simultánea de todos los hidrantes, determinando la altura manométrica y la eficiencia de bombeo que minimizan la ecuación 1. De esta primera simulación se obtiene, además, el hidrante crítico, que es el hidrante que recibe una presión menor. En la siguiente etapa se desactiva el hidrante crítico detectado en la primera simulación y se procede de la misma manera: con la demanda base de todos los hidrantes excepto el hidrante crítico se determina en EPANET la altura manométrica óptima que garantiza la presión requerida en todos los hidrantes operativos. A partir del caudal demandado y la altura manométrica determinada en EPANET, se obtiene de la matriz Q-H- $\eta$ la altura manométrica y la eficiencia de bombeo que minimizan la ecuación 1. Así, se obtiene un nuevo par de valores $\mathrm{Q}-\mathrm{H}$ en la estación de bombeo y el siguiente hidrante crítico. El procedimiento continúa con la desactivación del hidrante crítico detectado y una nueva simulación. De esta forma, en cada simulación, se detecta y desactiva un nuevo hidrante crítico y se obtiene una altura manométrica óptima.

El modelo se repite hasta un número de veces igual al número de hidrantes menos 1 , lo que permite determinar el número óptimo de hidrantes que no deben regar en las horas en las que el precio de la energía es mayor y la altura manométrica óptima cuando riega el grupo de hidrantes no identificados como críticos.

\section{3- Resultados y Discusión}

\section{1.- Modelización de la estación de bombeo}

La metodología descrita se ha aplicado a cada uno de los 11 sectores que conforman la comunidad de regantes del Bembézar Margen Derecha, aunque sólo los sectores IX (1262 ha) y X (1007 ha) se han seleccionado para el análisis de resultados.

La figura 2 muestra el resultado de la aplicación del modelo de simulación de estación de bombeo para los sectores IX (a) y X (b). El rango de operación de la estación de bombeo del sector IX se sitúa entre $36 \mathrm{~m}$ y $52 \mathrm{~m}$ con una demanda máxima de caudal de $1600 \mathrm{I} \mathrm{s}^{-1}$. El rango de operación de la estación de bombeo del sector X varía entre $36 \mathrm{~m} \mathrm{y}$ $50 \mathrm{~m}$ y el caudal máximo demandado en esta red es $1400 \mathrm{I} \mathrm{s}^{-1}$. En esta figura se observa que para caudales altos (superiores a $600 \mathrm{I} \mathrm{s}^{-1}$ ), las eficiencias de bombeo son altas para todo el rango de alturas manométricas analizadas. Sin embargo, el rendimiento es bajo cuando el caudal disminuye, aunque en ambos sectores se observan rendimientos en torno al $80 \%$ para caudales próximos a $200 \mathrm{I} \mathrm{s}^{-1}$. 

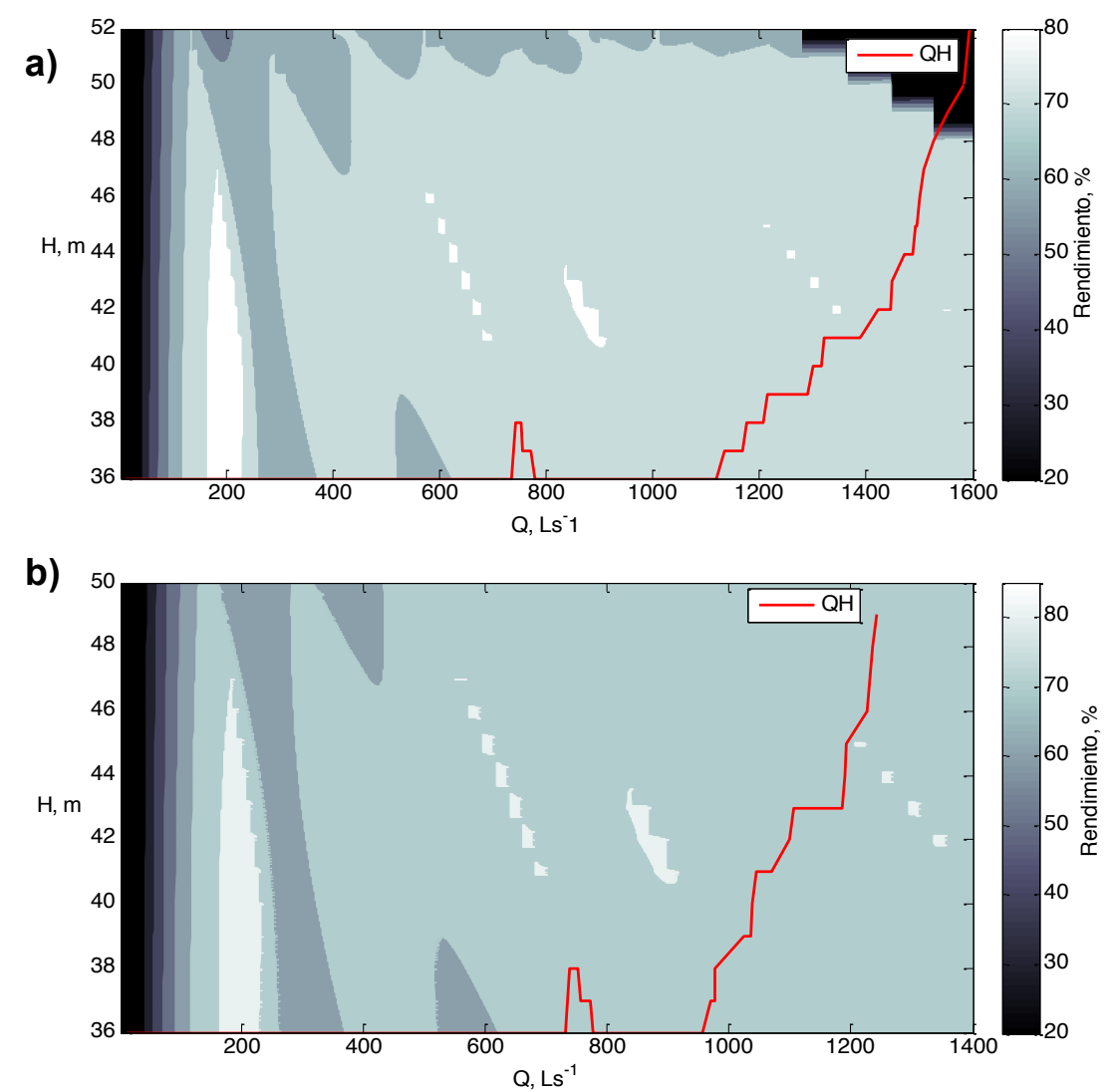

Figura 2. Modelización de la estación de bombeo de los sectores IX (a) y X (b) y curva Q-H obtenida.

\section{2.- Escenarios de gestión y estimación de ahorros}

La figura 3 muestra la altura manométrica óptima en función del número de hidrantes operativos para los sectores IX (a) y X (b).

En el sector IX (Figura 3.a) se observa una reducción importante de la altura manométrica, desde $48 \mathrm{~m}$ hasta alcanzar un valor mínimo de $36 \mathrm{~m}$ tras desactivar 42 hidrantes. El valor de $48 \mathrm{~m}$ se corresponde con la altura manométrica óptima para el escenario A. Sin embargo, en este sector no es posible la operación simultánea de todos los hidrantes ya que la estación de bombeo no es capaz de suministrar el par de valores Q-H requerido para un $100 \%$ de simultaneidad. Esto se observa en la figura 2.a, en la cual la línea que representa la curva Q-H se encuentra en una zona de colores oscuros (parte superior derecha) cuando se considera que todos los hidrantes están abiertos. En este caso el escenario A implica el funcionamiento de todos los hidrantes excepto 4 hidrantes críticos. En este escenario, la estación de bombeo trabaja con una eficiencia de bombeo del $75 \%$ y el consumo de energía por unidad de volumen de agua aplicada es $0.174 \mathrm{kWh} \mathrm{m}^{-3}$ (Figura 3.a).

El escenario B contempla la operación de todos los hidrantes salvo los 42 hidrantes críticos detectados. En este caso, la altura manométrica óptima es $36 \mathrm{~m}$, lo que se corresponde con una eficiencia en la estación de bombeo del $75 \%$. En este escenario, la 
energía consumida por unidad de agua aplicada se estima en $0.133 \mathrm{kWh} \mathrm{m}^{-3}$ (Figura 3.a). Por tanto, el escenario B supone un ahorro del $23.2 \%$ con respecto al escenario $\mathrm{A}$.

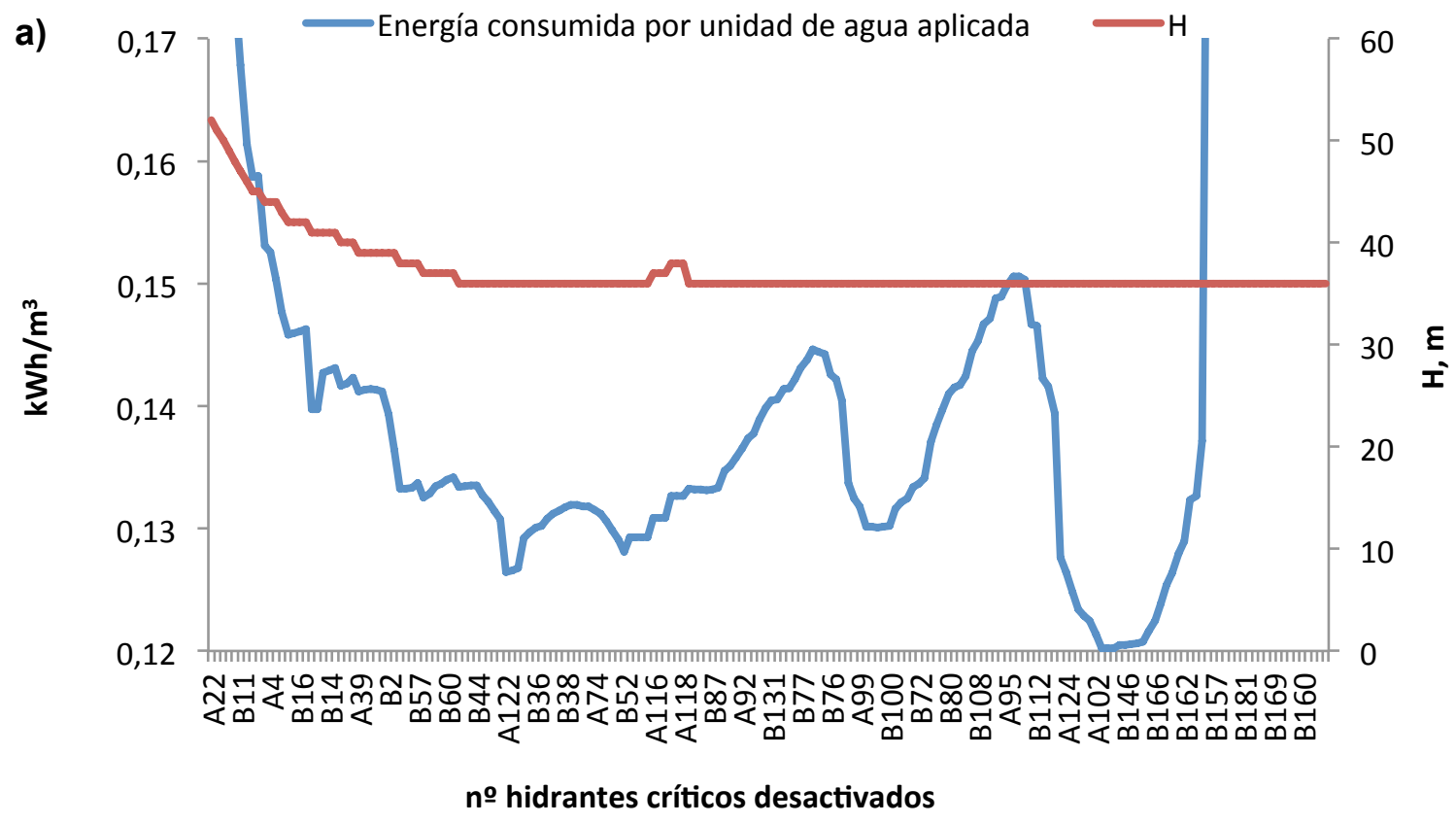

b)

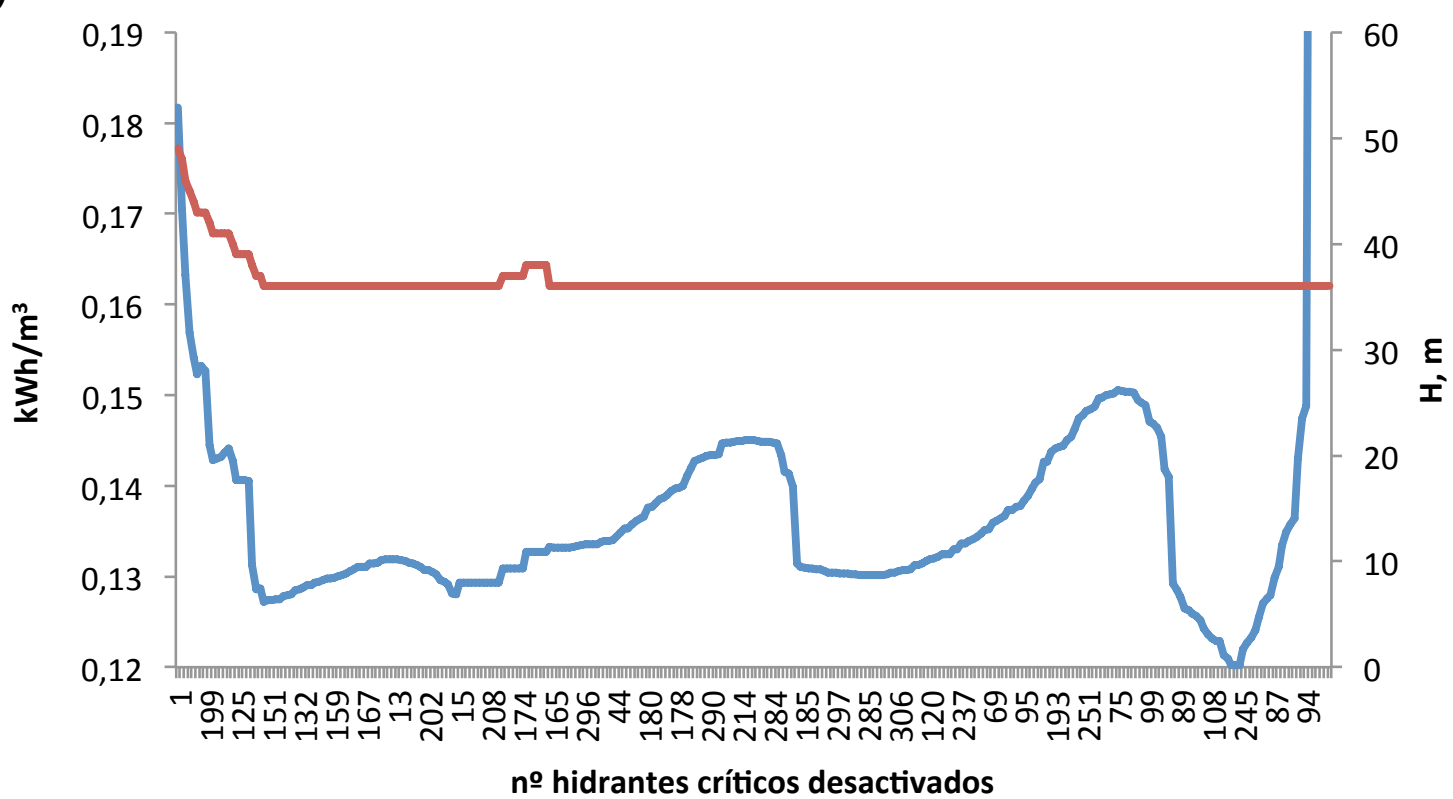

Figura 3. Energía consumida por unidad de agua aplicada y altura manométrica óptima en función del número de hidrantes críticos desactivados en los sectores IX (a) y X (b).

En el sector $X$, el valor mínimo de altura manométrica, $36 \mathrm{~m}$, se alcanza tras desactivar 22 hidrantes críticos (Figura 3.b). En el escenario A, la altura manométrica óptima es $49 \mathrm{~m}$, alcanzando en estas condiciones una eficiencia en el bombeo del $73 \%$ (Figura 2.b). En el escenario B, la altura manométrica óptima cuando los 22 hidrantes críticos detectados no están funcionando es $36 \mathrm{~m}$, lo que conlleva un rendimiento del $77 \%$. El consumo de energía por unidad de volumen de agua aplicada en el escenario A es 0.182 
$\mathrm{kWh} \mathrm{m}{ }^{-3}$ mientras que en el escenario $\mathrm{B}$ es $0.127 \mathrm{kWh} \mathrm{m}^{-3}$, lo que supone un ahorro del 25.8 $\%$.

El escenario B en ambos sectores contempla la adopción de la altura manométrica óptima en la estación de bombeo y la desactivación de 42 hidrantes (sector IX) y 22 hidrantes (sector X). Sin embargo, es posible seleccionar otras estrategias en las que la altura manométrica óptima no sea la mínima detectada pero no sea necesario desactivar todos los hidrantes críticos, lo que se correspondería con valores intermedios entre el escenario A y el B.

\section{4.- Conclusiones}

El aumento de las necesidades energéticas en las redes de riego a presión, junto con el incremento en el precio de este recurso obliga al desarrollo de estrategias que permitan reducir el consumo y el coste de energía.

En este trabajo se ha propuesto una estrategia de minimización de las necesidades energéticas en redes de riego definiendo dos escenarios de gestión: escenario $A$, en el que se determina la altura manométrica óptima en la estación de bombeo considerando un 100 $\%$ de simultaneidad de hidrantes; y escenario $B$, en el que se determina la altura manométrica óptima considerando que los hidrantes críticos no están activos. Ambos escenarios garantizan la presión de servicio en los hidrantes operativos. De esta forma, el escenario A sólo podría operar durante las horas en las que el precio de la energía es menor mientras que el escenario $B$ no tendría restricciones de funcionamiento.

La metodología propuesta se ha aplicado a los 11 sectores que conforman la comunidad de regantes de Bembézar Margen Derecha. En los dos sectores seleccionados como representativos, se ha obtenido una reducción en la altura manométrica óptima entre los escenarios A y B de $12 \mathrm{~m}$ y $13 \mathrm{~m}$ en los sectores IX y X, respectivamente, lo que se traduce en un ahorro potencial de $23.2 \%$ y $25.8 \%$ en los sectores IX y X.

Los dos escenarios propuestos suponen estrategias de gestión fáciles de adoptar: condicionar el funcionamiento de los hidrantes críticos para que operen en las horas en las que el precio de la energía es menor y flexibilizar la operación de aquellos hidrantes que no actúan como críticos para que puedan regar en cualquier momento del día.

\section{9- Bibliografía}

Carrillo Cobo M.T.. Rodríguez Díaz J.A.. Montesinos P., López Luque R.. Camacho Poyato E. (2011). Low energy consumption seasonal calendar for sectoring operation in pressurized irrigation networks. Irrigation Science 29 (2): 157-169.

FAO (2013) Afrontar la escasez de agua. Un marco de acción para la agricultura y la seguridad alimentaria. Organización de las Naciones Unidas para la Alimentación y la Agricultura, Roma.

Fernández García, I., Rodríguez Díaz J.A.. Montesinos P., Camacho Poyato E. (2014). Optimización del coste energético en redes de riego a presión con varios puntos de suministro. XXXII Congreso Nacional de Riegos. Madrid.

Jiménez Bello M.A., Martínez Alzamora F., Bou Soler V., Bartolí Ayala H.J. (2010) Methodology for grouping intakes of pressurised irrigation networks into sectors to minimise energy consumption. Biosyst Eng 105:429-438

Ministerio de Agricultura, Pesca y Alimentación, MAPA, (2001). Plan Nacional de Regadíos. Horizonte 2008. Madrid. 
Ministerio de Medio Ambiente y Medio Rural y Marino, MARM, (2006). Plan de Choque de Modernización de Regadíos. Madrid

Moreno M.A., Carrión P., Planells P., Ortega J.F., Tarjuelo J.M. (2007). Measurement and improvement of the energy efficiency at pumping stations. Biosyst. Eng. 98,479-486.

Pratap R. 2010. Getting started with Matlab. A quick introduction for Scientist and Engineers. Oxford University Press. USA.

Rodríguez Díaz J.A., Montesinos P., Camacho Poyato E. (2012). Detecting Critical Points in On-Demand Irrigation Pressurized Networks - A New Methodology. Water Resour Manage. DOI 10.1007/s11269-012-9981-8.

Rossman L.A. 2000. EPANET 2. Users manual. US Environmental Protection Agency (EPA). USA. 